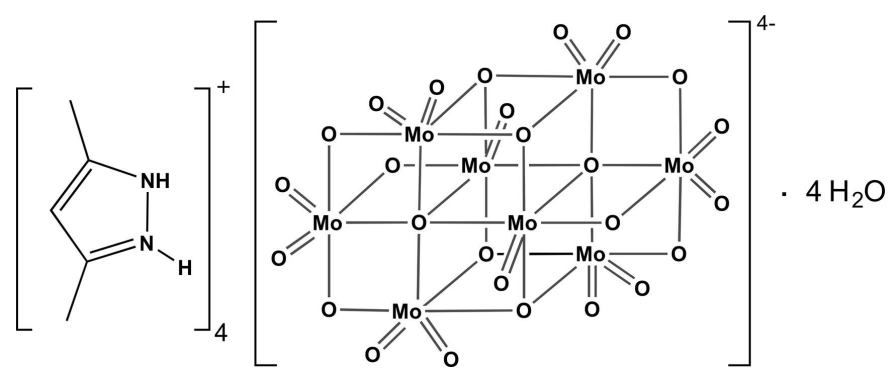

\title{
Redetermination of the crystal structure of 3,5-dimethylpyrazolium $\beta$-octa- molybdate tetrahydrate
}

Tatiana R. Amarante, Isabel S. Gonçalves and Filipe A. Almeida Paz*

Department of Chemistry, CICECO - Aveiro Institute of Materials, University of Aveiro, 3810-193 Aveiro, Portugal. *Correspondence e-mail: filipe.paz@ua.pt

Received 21 November 2015; accepted 29 November 2015

Edited by W. T. A. Harrison, University of Aberdeen, Scotland

The title compound, $\left(\mathrm{C}_{5} \mathrm{H}_{9} \mathrm{~N}_{2}\right)_{4}\left[\mathrm{Mo}_{8} \mathrm{O}_{26}\right] \cdot 4 \mathrm{H}_{2} \mathrm{O}$, was reported previously from a room-temperature data collection from which only the metal atoms could be refined anisotropically [FitzRoy et al. (1989). Inorg. Chim. Acta, 157, 187-194]. The current redetermination at 180 (2) K models all the non-H atoms with anisotropic displacement parameters and fully describes the supramolecular $\mathrm{N}-\mathrm{H} \cdots \mathrm{O}$ and $\mathrm{O}-\mathrm{H} \cdots \mathrm{O}$ hydrogen-bonded network connecting the 3,5-dimethylpyrazolium cations, the water molecules of crystallization and the $\beta$-octamolybdate anion. All $\mathrm{H}$ atoms involved in the three-dimensional hydrogen-bonding network could be located from difference Fourier maps, with the exception of those of one disordered water molecule, firstly seen in this structural report [refined over two distinct locations with siteoccupancy factors of 0.65 (2) and 0.35 (2)]. The complete $\beta$ octamolybdate anion is generated by a crystallographic inversion centre.

Keywords: crystal structure; 3,5-dimethylpyrazolium cations; octamolybdate( $(\mathrm{VI})$ anion; structure redetermination; hydrogen-bonding network.

CCDC reference: 1439409

\section{Related literature}

For the previous determination of the title compound at room temperature (Cambridge Structural Database refcode: JAMFEI), see: FitzRoy et al. (1989). For a description of the Cambridge Structural Database, see: Groom \& Allen (2014). For previous studies investigating recovered molybdenum(VI) catalysts, see: Amarante et al. (2015); Lysenko et al. (2015).

\section{Experimental}

\subsection{Crystal data}

$\left(\mathrm{C}_{5} \mathrm{H}_{9} \mathrm{~N}_{2}\right)_{4}\left[\mathrm{Mo}_{8} \mathrm{O}_{26}\right] \cdot 4 \mathrm{H}_{2} \mathrm{O}$

$M_{r}=1644.15$

Triclinic, $P \overline{1}$

$a=10.1105$ (9) $\AA$

$b=10.7469$ (9) $\AA$

$c=11.9839(10) \AA$

$\alpha=64.103$ (3)

$\beta=84.272(3)^{\circ}$

\subsection{Data collection}

Bruker D8 QUEST diffractometer Absorption correction: multi-scan (SADABS; Bruker, 2001) $T_{\min }=0.663, T_{\max }=0.745$

\subsection{Refinement}

$R\left[F^{2}>2 \sigma\left(F^{2}\right)\right]=0.026$

$w R\left(F^{2}\right)=0.056$

$S=1.06$

4141 reflections

320 parameters

7 restraints

Table 1

Hydrogen-bond geometry $\left(\AA,^{\circ}\right)$.

\begin{tabular}{lllll}
\hline$D-\mathrm{H} \cdots A$ & $D-\mathrm{H}$ & $\mathrm{H} \cdots A$ & $D \cdots A$ & $D-\mathrm{H} \cdots A$ \\
\hline $\mathrm{N} 1-\mathrm{H} 1 \cdots \mathrm{O} 2 W^{\mathrm{i}}$ & 0.94 & 1.77 & $2.689(7)$ & 164 \\
$\mathrm{~N} 2-\mathrm{H} 2 \cdots \mathrm{O} 1 W$ & 0.95 & 1.84 & $2.776(5)$ & 171 \\
$\mathrm{~N} 3-\mathrm{H} 3 \cdots \mathrm{O} 13^{\mathrm{ii}}$ & 0.95 & 2.28 & $2.869(5)$ & 120 \\
$\mathrm{~N} 4-\mathrm{H} 4 \cdots \mathrm{O} 10$ & 0.95 & 1.93 & $2.801(4)$ & 152 \\
$\mathrm{O} 1 W-\mathrm{H} 1 X \cdots \mathrm{O} 5^{\text {iii }}$ & 0.95 & 1.88 & $2.785(4)$ & 160 \\
$\mathrm{O} 1 W-\mathrm{H} 1 Y \cdots \mathrm{O} 3$ & 0.94 & 1.91 & $2.848(4)$ & 172 \\
\hline
\end{tabular}

Symmetry codes: (i) $x, y, z+1$; (ii) $-x,-y+1,-z+1$; (iii) $-x+1,-y+1,-z+2$.

Data collection: APEX2 (Bruker, 2012); cell refinement: SAINT (Bruker, 2012); data reduction: $S A I N T$; $\operatorname{program}(\mathrm{s})$ used to solve structure: SHELXS97 (Sheldrick, 2008); program(s) used to refine structure: SHELXL2014 (Sheldrick, 2015); molecular graphics: DIAMOND (Brandenburg, 1999); software used to prepare material for publication: SHELXL2014.

\section{Acknowledgements}

Funding Sources and Entities Fundação para a Ciência e a Tecnologia (FCT, Portugal), the European Union, QREN, 
FEDER through Programa Operacional Factores de Competitividade (COMPETE), CICECO-Aveiro Institute of Materials (Ref. FCT UID/CTM/50011/2013) financed by national funds through the FCT/MEC and when applicable co-financed by FEDER under the PT2020 Partnership Agreement.

Projects and Individual grants We wish to thank FCT for funding the R\&D project FCOMP-01-0124-FEDER-041282 (Ref. FCT EXPL/CTM-NAN/0013/2013), and also CICECO for specific funding towards the purchase of the single-crystal diffractometer. FCT is gratefully acknowledged for the postdoctoral research grant No. SFRH/BPD/97660/2013 (to TRA).

Supporting information for this paper is available from the IUCr electronic archives (Reference: HB7547).

\section{References}

Amarante, T. R., Antunes, M. M., Valente, A. A., Almeida Paz, F. A., Pillinger, M. \& Gonçalves, I. S. (2015). Inorg. Chem. 54, 9690-9703.

Brandenburg, K. (1999). DIAMOND. Crystal Impact GbR, Bonn, Germany. Bruker (2001). SADABS. Bruker AXS Inc., Madison, Wisconsin, USA.

Bruker (2012). SAINT and APEX2. Bruker AXS Inc., Madison, Wisconsin, USA.

FitzRoy, M. D., Fallon, M. D. \& Murray, K. S. (1989). Inorg. Chim. Acta, 157, 187-194.

Groom, C. R. \& Allen, F. H. (2014). Angew. Chem. Int. Ed. 53, 662-671.

Lysenko, A. B., Senchyk, G. A., Domasevitch, K. V., Hauser, J., Fuhrmann, D., Kobalz, M., Krautscheid, H., Neves, P., Valente, A. A. \& Gonçalves, I. S. (2015). Inorg. Chem. 54, 8327-8338.

Sheldrick, G. M. (2008). Acta Cryst. A64, 112-122.

Sheldrick, G. M. (2015). Acta Cryst. C71, 3-8. 


\title{
supporting information
}

Acta Cryst. (2015). E71, m244-m245 [https://doi.org/10.1107/S2056989015022823]

\section{Redetermination of the crystal structure of 3,5-dimethylpyrazolium $\beta$ - octamolybdate tetrahydrate}

\author{
Tatiana R. Amarante, Isabel S. Gonçalves and Filipe A. Almeida Paz
}

\section{S1. Context and introduction}

Research efforts in the development and application of new hybrid molybdenum(vi) heterogeneous catalysts require on a daily basis the recovery of used materials and their characterisation in the solid state to check for structural modifications of the employed compound (Amarante et al., 2015; Lysenko et al., 2015). Often, the use of drastic experimental conditions leads to the formation of secondary products, which crystallise in the medium as trace amounts of impurity compounds. It is, thus, imperative that most of these possible products are fully described in the solid state in the most accurate fashion.

The title compound, $\left(\mathrm{C}_{5} \mathrm{H}_{9} \mathrm{~N}_{2}\right)_{4}\left[\mathrm{Mo}_{8} \mathrm{O}_{26}\right] \cdot 4\left(\mathrm{H}_{2} \mathrm{O}\right)$, was previously reported by FitzRoy et al. (1989). Besides the fact that the authors did not fully elucidate the hydrogen bonding network of this material (hydrogen atoms were placed geometrically) and that only the metallic centers could be refined anisotropically (from a room-temperature determination), the unit cell parameters reported in the main text and in the abstract do not match (viz. the $c$ axis length). A search and match at the Cambridge Structural Database (Groom et al., 2014) also seems to ignore the presence of $\mathrm{Mo}(\mathrm{VI})$ metal centers. In this context, we decided to recollect the crystal structure of the title compound at low temperature to fully elucidate its finer structural details.

\section{S2. Structure description}

The asymmetric unit of the title compound is composed of two 3,5-dimethylpyrazolium cations, $\left(\mathrm{C}_{5} \mathrm{H}_{9} \mathrm{~N}_{2}\right)^{+}$, one half of the $\beta$-octamolybdate anion, $\beta$-[ $\left[\mathrm{Mo}_{8} \mathrm{O}_{26}\right]^{4}$, and two water molecules of crystallisation. Noteworthy, while one water molecule was fully located in its crystallographic position and even the associated hydrogen atoms found from difference Fourier maps, the other was found to be disordered over two distinct locations, O2W and O3W (Fig. 1). This feature was not disclosed in the previous structural determination by FitzRoy et al. (1989).

The molecular geometrical parameters for the $\beta$-octamolybdate anion are typical, exhibiting the usual four families of Mo-O bonds: Mo- $-t$ to terminal oxido groups [bond distances in the 1.691 (3)-1.715 (3) $\AA$ range]; Mo- $-\mathrm{O} b$ to $\mu_{2^{-}}$ bridging oxido groups [bond distances in the 1.754 (3)-2.268 (3) $\AA$ range]; $\mathrm{Mo}-\mathrm{O} c$ to $\mu_{3}$-bridging oxido groups [bond distances in the 1.953 (3)-2.351 (3) $\AA$ range]; $\mathrm{Mo}-\mathrm{O} c$ to $\mu_{5}$-bridging oxido groups [bond distances in the 2.146 (3)-2.435 (3) $\AA$ range]. The four crystallographically independent Mo(VI) metal centers are thus hexacoordinated in a typical $\left\{\mathrm{MoO}_{6}\right\}$ fashion resembling highly distorted octahedra: while this trans internal $\mathrm{O}-\mathrm{Mo}-\mathrm{O}$ octahedral angles were found in the 145.29 (11)-173.17 (12) ${ }^{\circ}$ range, the $c i s$ angles refined instead in the 70.01 (9)-105.85 (14) ${ }^{\circ}$ interval. We note that this wide dispersion for the internal octahedral angles is a notable and well-known consequence of the marked trans effect created by the terminal oxido groups which displace the metal centers from the center of the octahedra. 
The $\beta$-octamolybdate anion, located in the center of the unit cell, interacts with the remaining chemical species through a series of both electrostatic interactions and hydrogen bonds (Fig. 2). One crystallographically independent 3,5-di-

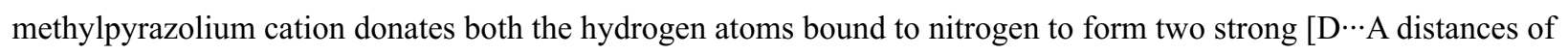
2.801 (4) and $2.869(5) \AA]$ and relatively directional [ $<$ (DHA) angles of 120 and $152^{\circ}$ ] charged $\mathrm{N}-\mathrm{H} \cdots \mathrm{O}$ interactions with the polyoxoanion. The other cation has, however, a completely distinct behaviour: the same hydrogen atoms are instead donated in similar (strong and highly directional) interactions with neighbouring water molecules: while the $\mathrm{D} \cdots \mathrm{A}$ distances are 2.689 (7) and 2.776 (5) $\AA$, the $<$ (DHA) interaction angles are close to linearity, being 171 and $164^{\circ}$. The water molecules are instead interacting with the $\beta$-octamolybdate anion as depicted in both Figs. 1 and 2. Indeed, as depicted in Fig. 3 the water molecules play a decisive role in the overall crystal packing, acting as molecular fillers to effectively occupy the available space left from the arrangement of inorganic anions and organic cations.

\section{S3. Synthesis and crystallization}

All chemicals were purchased from commercial sources and used as received without additional purification steps.

A Teflon-lined stainless steel vessel was charged with a reaction mixture composed of $\mathrm{MoO}_{3}(0.34 \mathrm{~g}, 2.43 \mathrm{mmol}), 3,5-$ dimethylpyrazole $(0.11 \mathrm{~g}, 1.21 \mathrm{mmol})$ and water $(c a .25 \mathrm{~mL})$ and heated in an oven at $160{ }^{\circ} \mathrm{C}$ for $26 \mathrm{~h}$. The resultant blueish solid was filtered from the aqueous mother liquor and washed with an excess of water and $(4 \times 10 \mathrm{~mL})$ diethyl ether, dried at ambient temperature and characterized in the solid state. Colourless plates of the title compound were directly harvested from the walls of the Teflon vessel.

Selected FT—IR $\left(\mathrm{KBr}, \mathrm{cm}^{-1}\right): \tilde{\mathrm{n}}=948(v s), 910(v s), 843(s), 733(s), 714(s), 663(s)$.

\section{S4. Refinement details}

Hydrogen atoms bound to carbon atoms were placed at idealized positions with $\mathrm{C}-\mathrm{H}=0.95$ or $0.98 \AA$ (for the aromatic and methyl groups, respectively), and included in the final structural model in riding-motion approximation with the isotropic thermal displacement parameters fixed at 1.2 or $1.5 \times U_{\text {eq }}$, respectively, of the carbon atom to which they are attached.

Hydrogen atoms associated with nitrogen atoms have been directly located from difference Fourier maps and were included in the model with the $\mathrm{N}-\mathrm{H}$ distances restrained to 0.95 (1) $\AA$ in order to ensure a chemically reasonable environment for these moieties. These hydrogen atoms were modelled with the isotropic thermal displacement parameters fixed at $1.5 \times U_{\text {eq }}(\mathrm{N})$.

A total of two water molecules of crystallisation were directly located from difference Fourier maps. Though O1W was included in the final structural model by assuming full site occupancy and a typical anisotropic displacement behaviour, the second molecule was found to be disordered over two close crystallographic positions: $\mathrm{O} 2 \mathrm{~W}$ and $\mathrm{O} 3 \mathrm{~W}$. These species were included in the structural model with linked site occupancy [which ultimately refined to 0.65 (2) and 0.35 (2), respectively] and by assuming an independent isotropic displacement behaviour. For O1W the two hydrogen atoms were markedly visible in difference Fourier maps and were included in the final model with the $\mathrm{O}-\mathrm{H}$ and $\mathrm{H} \cdots \mathrm{H}$ distances restrained to 0.95 (1) and 1.55 (1) $\AA$, respectively, in order to ensure a chemically reasonable geometry for this molecule. These hydrogen atoms were modelled with the isotropic thermal displacement parameters fixed at $1.5 \times U_{\text {eq }}(\mathrm{O} 1 \mathrm{~W})$. 


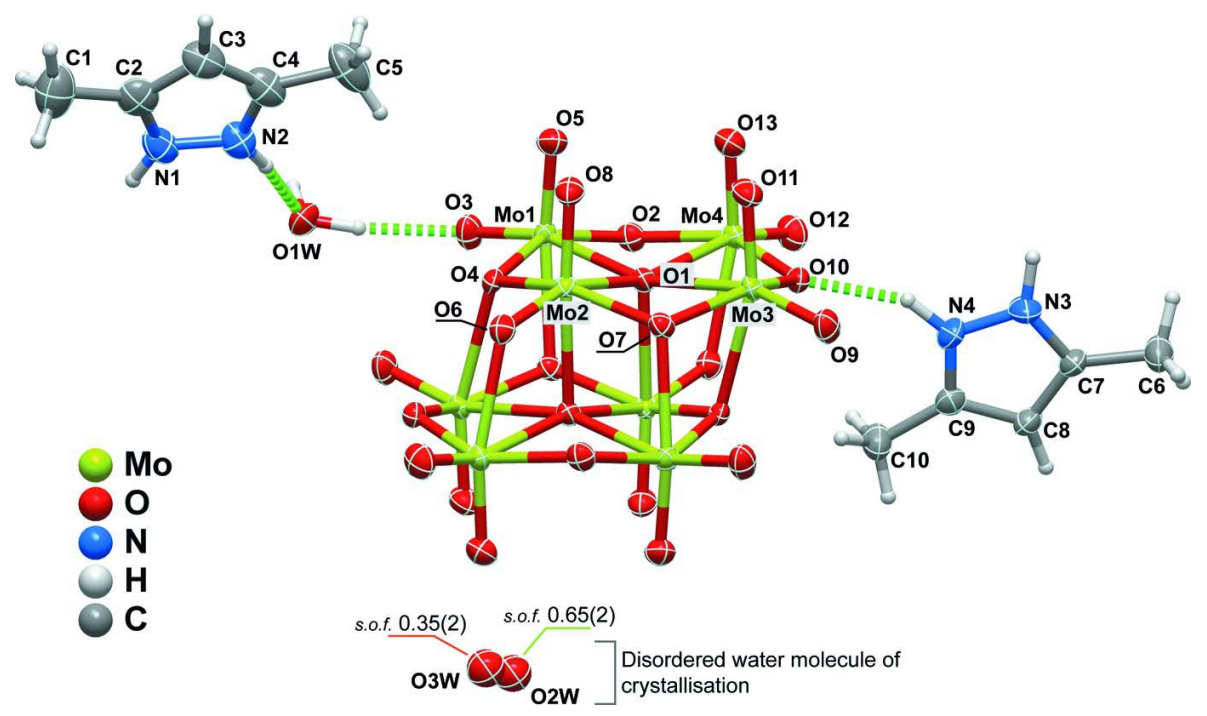

Figure 1

Schematic representation of the molecular entities composing the asymmetric unit of the title compound. The $\beta$ octamolybdate anion has been completed by inversion symmetry for the sake of chemical accuracy. All non-hydrogen atoms are represented as displacement ellipsoids drawn at the $60 \%$ probability level and hydrogen atoms as small spheres with arbitrary radii. Non-hydrogen atoms belonging to the asymmetric unit have been labelled for clarity. Dashed green broken lines indicate $\mathrm{N}-\mathrm{H} \cdots \mathrm{O}$ and $\mathrm{O}-\mathrm{H} \cdots \mathrm{O}$ hydrogen-bonding interactions (see Table for geometrical details). 

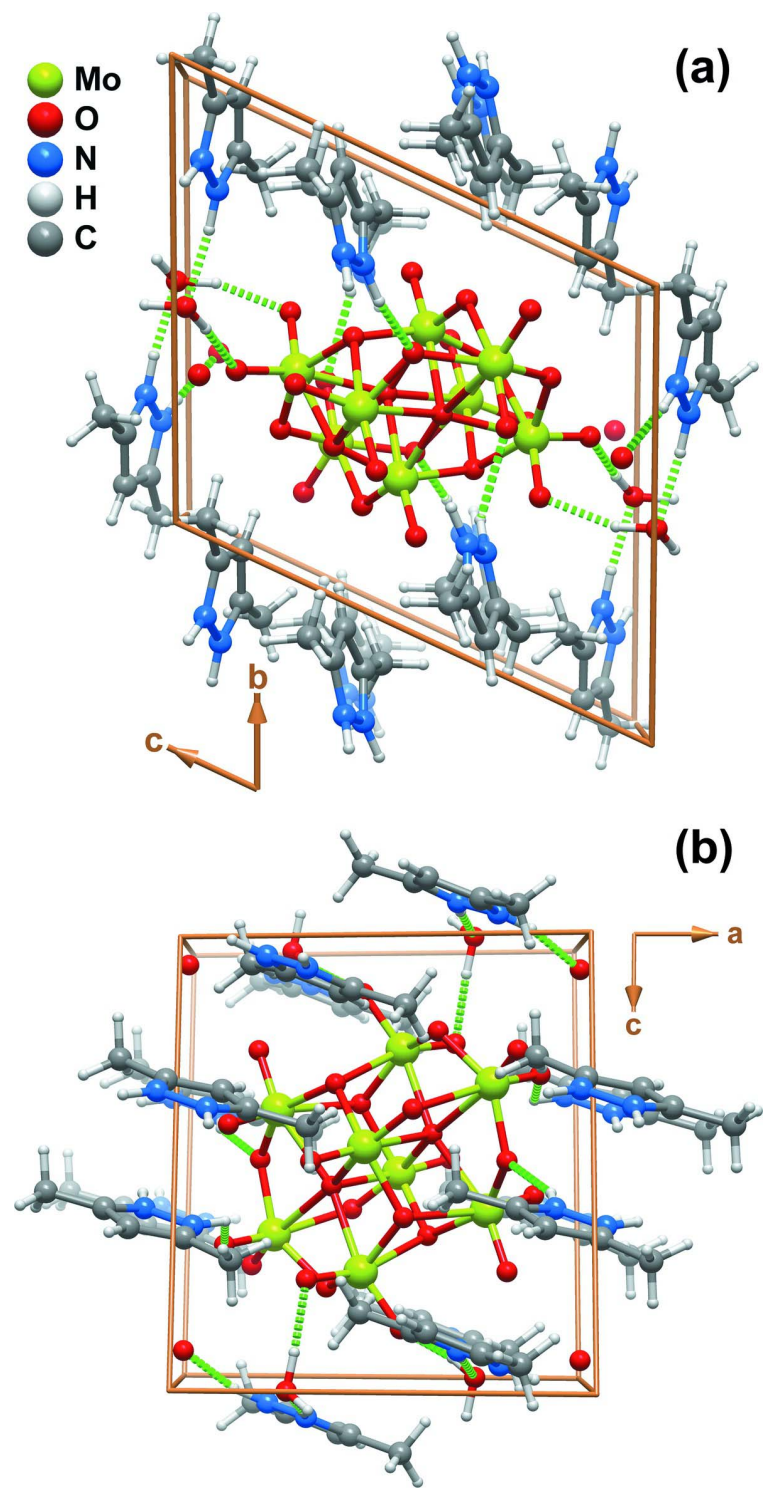

Figure 2

Crystal packing of the title compound viewed in perspective along the (a) [100] (b) [010] directions of the unit cell emphasising the supramolecular $\mathrm{N}-\mathrm{H} \cdots \mathrm{O}$ and $\mathrm{O}-\mathrm{H} \cdots \mathrm{O}$ hydrogen-bonding interactions (dashed green lines) interconnecting the three types of chemical species present in the crystal structure of the title compound. 


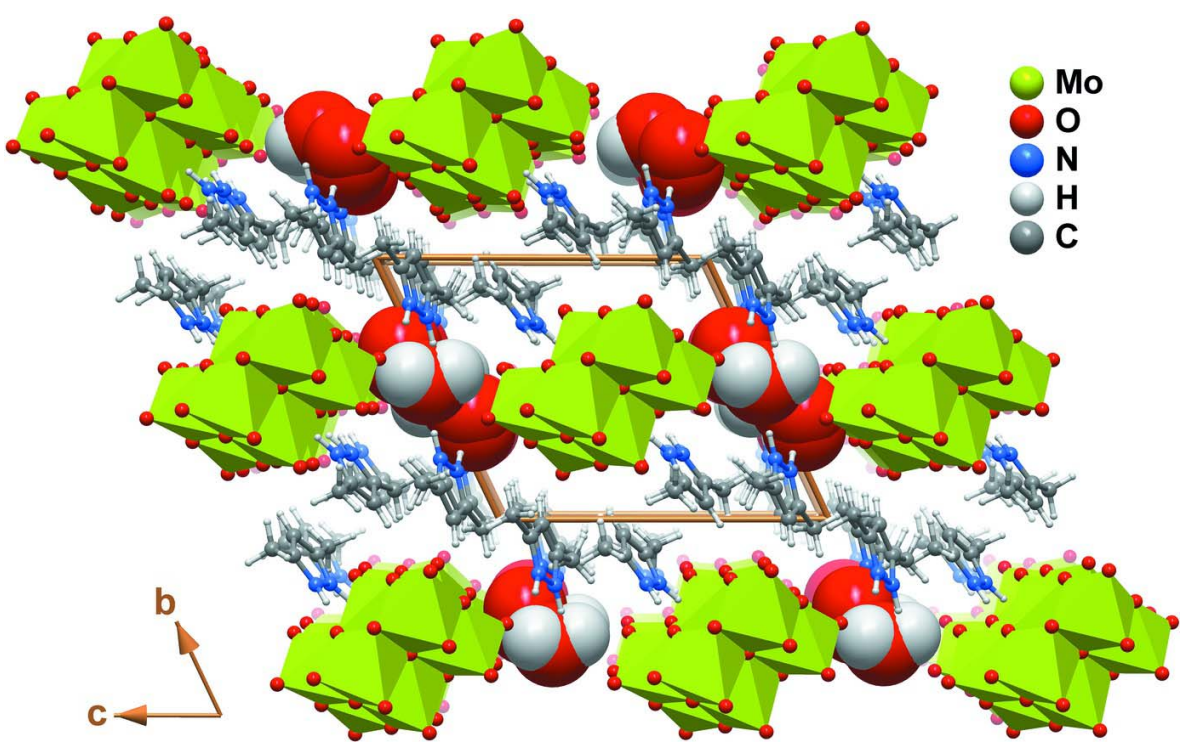

Figure 3

Mixed polyhedral (for the $\beta$-octamolybdate anion), ball-and-stick (for the 3,5-dimethylpyrazolium cations) and space filling (for the water molecules of crystallisation) schematic representation of the crystal packing of the title compound viewed in perspective along the [100] direction of the unit cell. The Figure illustrates well how the inorganic component of the crystal structure is embedded into an organic matrix, with the entrapped water molecules of crystallization acting as molecular fillers interacting with the hybrid network through hydrogen bonds.

Tetrakis(3,5-dimethylpyrazolium) $\beta$-octamolybdate tetrahydrate

\section{Crystal data}

$\left(\mathrm{C}_{5} \mathrm{H}_{9} \mathrm{~N}_{2}\right)_{4}\left[\mathrm{Mo}_{8} \mathrm{O}_{26}\right] \cdot 4 \mathrm{H}_{2} \mathrm{O}$

$M_{r}=1644.15$

Triclinic, $P \overline{1}$

$a=10.1105(9) \AA$

$b=10.7469(9) \AA$

$c=11.9839(10) \AA$

$\alpha=64.103(3)^{\circ}$

$\beta=84.272(3)^{\circ}$

$\gamma=75.826(3)^{\circ}$

$V=1135.67(17) \AA^{3}$

\section{Data collection}

\section{Bruker D8 QUEST}

diffractometer

Radiation source: Sealed tube

Multi-layer X-ray mirror monochromator

Detector resolution: 10.4167 pixels $\mathrm{mm}^{-1}$

$\omega / \varphi$ scans

Absorption correction: multi-scan

(SADABS; Bruker, 2001)

$T_{\min }=0.663, T_{\max }=0.745$
$Z=1$

$F(000)=796$

$D_{\mathrm{x}}=2.404 \mathrm{Mg} \mathrm{m}^{-3}$

Mo $K \alpha$ radiation, $\lambda=0.71073 \AA$

Cell parameters from 9938 reflections

$\theta=2.6-25.4^{\circ}$

$\mu=2.24 \mathrm{~mm}^{-1}$

$T=180 \mathrm{~K}$

Plate, colourless

$0.20 \times 0.14 \times 0.01 \mathrm{~mm}$

25625 measured reflections

4141 independent reflections

3175 reflections with $I>2 \sigma(I)$

$R_{\text {int }}=0.042$

$\theta_{\max }=25.4^{\circ}, \theta_{\min }=3.7^{\circ}$

$h=-12 \rightarrow 12$

$k=-12 \rightarrow 12$

$l=-14 \rightarrow 14$ 


\section{Refinement}

Refinement on $F^{2}$

Least-squares matrix: full

$R\left[F^{2}>2 \sigma\left(F^{2}\right)\right]=0.026$

$w R\left(F^{2}\right)=0.056$

$S=1.06$

4141 reflections

320 parameters

7 restraints
Hydrogen site location: mixed

$\mathrm{H}$ atoms treated by a mixture of independent and constrained refinement

$w=1 /\left[\sigma^{2}\left(F_{\mathrm{o}}^{2}\right)+(0.0188 P)^{2}+2.2481 P\right]$

where $P=\left(F_{\mathrm{o}}^{2}+2 F_{\mathrm{c}}{ }^{2}\right) / 3$

$(\Delta / \sigma)_{\max }=0.001$

$\Delta \rho_{\max }=0.70 \mathrm{e} \AA^{-3}$

$\Delta \rho_{\min }=-0.54$ e $\AA^{-3}$

Special details

Geometry. All esds (except the esd in the dihedral angle between two 1.s. planes) are estimated using the full covariance matrix. The cell esds are taken into account individually in the estimation of esds in distances, angles and torsion angles; correlations between esds in cell parameters are only used when they are defined by crystal symmetry. An approximate (isotropic) treatment of cell esds is used for estimating esds involving l.s. planes.

Fractional atomic coordinates and isotropic or equivalent isotropic displacement parameters $\left(\AA^{2}\right)$

\begin{tabular}{|c|c|c|c|c|c|}
\hline & $x$ & $y$ & $z$ & $U_{\text {iso }} * / U_{\text {eq }}$ & Occ. $(<1)$ \\
\hline Mo1 & $0.44983(3)$ & $0.44979(4)$ & $0.75253(3)$ & $0.01430(9)$ & \\
\hline Mo2 & 0.45658 & $0.68126(4)$ & $0.47103(3)$ & $0.01229(9)$ & \\
\hline Mo3 & 0.23903 & $0.56541(4)$ & $0.38528(3)$ & $0.01415(9)$ & \\
\hline Mo4 & $0.23047(3)$ & $0.32798(4)$ & $0.67012(3)$ & $0.01511(9)$ & \\
\hline $\mathrm{O} 1$ & 0.3753 & $0.4944(3)$ & $0.5589(2)$ & $0.0142(6)$ & \\
\hline $\mathrm{O} 2$ & 0.3635 & 0.3005 & $0.7899(2)$ & $0.0177(6)$ & \\
\hline $\mathrm{O} 3$ & $0.5489(3)$ & $0.3881(3)$ & 0.8808 & $0.0235(7)$ & \\
\hline $\mathrm{O} 4$ & $0.5539(3)$ & $0.5839(3)$ & $0.6298(2)$ & $0.0145(6)$ & \\
\hline $\mathrm{O} 5$ & $0.3166(3)$ & $0.5748(3)$ & 0.7683 & $0.0208(7)$ & \\
\hline O6 & 0.5740 & 0.7845 & $0.3880(2)$ & $0.0168(6)$ & \\
\hline $\mathrm{O} 7$ & 0.3838 & 0.6770 & $0.3281(2)$ & $0.0153(6)$ & \\
\hline O8 & 0.3261 & 0.7924 & 0.5040 & $0.0190(6)$ & \\
\hline O9 & 0.1894 & 0.5797 & $0.2484(3)$ & $0.0232(7)$ & \\
\hline $\mathrm{O} 10$ & $0.1936(3)$ & $0.3896(3)$ & $0.4970(2)$ & $0.0160(6)$ & \\
\hline O11 & $0.1191(3)$ & $0.6842(3)$ & 0.4197 & $0.0220(7)$ & \\
\hline $\mathrm{O} 12$ & $0.1720(3)$ & $0.1773(3)$ & $0.7422(3)$ & $0.0255(7)$ & \\
\hline $\mathrm{O} 13$ & $0.1090(3)$ & $0.4568(3)$ & 0.6928 & $0.0213(7)$ & \\
\hline N1 & $0.7819(4)$ & $0.8121(5)$ & $0.9458(3)$ & $0.0294(9)$ & \\
\hline H1 & $0.865(3)$ & $0.747(4)$ & $0.979(4)$ & $0.044^{*}$ & \\
\hline $\mathrm{N} 2$ & 0.6839 & $0.7657(4)$ & $0.9163(4)$ & $0.0281(9)$ & \\
\hline $\mathrm{H} 2$ & $0.692(5)$ & 0.6692 (19) & $0.934(5)$ & $0.042 *$ & \\
\hline N3 & $-0.0378(4)$ & $0.2498(4)$ & $0.3524(4)$ & $0.0295(9)$ & \\
\hline $\mathrm{H} 3$ & $-0.116(3)$ & $0.314(4)$ & $0.362(5)$ & $0.044^{*}$ & \\
\hline N4 & $0.0827(4)$ & $0.2507(4)$ & $0.3913(4)$ & $0.0289(9)$ & \\
\hline $\mathrm{H} 4$ & $0.090(5)$ & $0.314(4)$ & $0.425(4)$ & $0.043 *$ & \\
\hline $\mathrm{C} 1$ & $0.8229(6)$ & $1.0327(6)$ & $0.9381(5)$ & $0.0491(15)$ & \\
\hline H1A & 0.8535 & 0.9870 & 1.0247 & $0.074 *$ & \\
\hline H1B & 0.7696 & 1.1293 & 0.9183 & $0.074 *$ & \\
\hline $\mathrm{H} 1 \mathrm{C}$ & 0.9023 & 1.0365 & 0.8837 & $0.074 *$ & \\
\hline $\mathrm{C} 2$ & $0.7364(5)$ & $0.9494(5)$ & $0.9191(4)$ & 0.0319 (11) & \\
\hline
\end{tabular}




\begin{tabular}{lllll} 
C3 & $0.6048(5)$ & $0.9913(5)$ & $0.8718(4)$ & $0.0316(11)$ \\
H3A & 0.5471 & 1.0837 & 0.8443 & $0.038^{*}$ \\
C4 & $0.5743(5)$ & $0.8733(5)$ & $0.8726(4)$ & $0.0312(11)$ \\
C5 & $0.4486(5)$ & $0.8533(6)$ & $0.8341(5)$ & $0.0420(14)$ \\
H5A & 0.4728 & 0.7778 & 0.8058 & $0.063^{*}$ \\
H5B & 0.4035 & 0.9420 & 0.7663 & $0.063^{*}$ \\
H5C & 0.3866 & 0.8271 & 0.9047 & $0.063^{*}$ \\
C6 & $-0.1363(5)$ & $0.1101(5)$ & $0.2791(5)$ & $0.0329(12)$ \\
H6A & -0.2222 & 0.1413 & 0.3150 & $0.049^{*}$ \\
H6B & -0.1209 & 0.0082 & 0.3021 & $0.049^{*}$ \\
H6C & -0.1412 & 0.1624 & 0.1885 & $0.049^{*}$ \\
C7 & $-0.0218(4)$ & $0.1381(5)$ & $0.3270(4)$ & $0.0208(10)$ \\
C8 & $0.1130(4)$ & $0.0663(5)$ & $0.3502(4)$ & $0.0225(10)$ \\
H8 & 0.1545 & -0.0174 & 0.3399 & $0.027^{*}$ \\
C9 & $0.1768(4)$ & $0.1398(5)$ & $0.3917(4)$ & $0.0208(10)$ \\
C10 & $0.3202(4)$ & $0.1135(5)$ & $0.4290(5)$ & $0.0303(11)$ \\
H10A & 0.3497 & 0.2034 & 0.3936 & $0.045^{*}$ \\
H10B & 0.3787 & 0.0479 & 0.3983 & $0.045^{*}$ \\
H10C & 0.3268 & 0.0717 & 0.5196 & $0.045^{*}$ \\
O1W & $0.7299(3)$ & $0.4764(4)$ & $0.9840(3)$ & $0.0276(7)$ \\
H1X & $0.711(5)$ & $0.439(5)$ & $1.0698(12)$ & $0.041^{*}$ \\
H1Y & $0.676(4)$ & $0.449(5)$ & $0.943(3)$ & $0.041^{*}$ \\
O2W & $0.9897(6)$ & $0.5952(10)$ & $0.0682(5)$ & $0.031(2)^{*}$ \\
O3W & $1.0101(11)$ & $0.6562(19)$ & $0.0507(10)$ & $0.033(4)^{*}$ \\
& & & & $0.65(2)$ \\
\hline
\end{tabular}

Atomic displacement parameters $\left(\AA^{2}\right)$

\begin{tabular}{lllllll}
\hline & $U^{11}$ & $U^{22}$ & $U^{33}$ & $U^{12}$ & $U^{13}$ & $U^{23}$ \\
\hline Mo1 & $0.01445(18)$ & $0.0179(2)$ & $0.01272(18)$ & $-0.00473(15)$ & $0.00061(14)$ & $-0.00798(16)$ \\
Mo2 & $0.01153(18)$ & $0.01158(19)$ & $0.01458(18)$ & $-0.00190(14)$ & $-0.00116(14)$ & $-0.00643(15)$ \\
Mo3 & $0.01088(18)$ & $0.0166(2)$ & $0.01578(19)$ & $-0.00309(15)$ & $-0.00217(14)$ & $-0.00718(16)$ \\
Mo4 & $0.01333(18)$ & $0.0163(2)$ & $0.01719(19)$ & $-0.00532(15)$ & $0.00086(14)$ & $-0.00759(16)$ \\
O1 & $0.0124(14)$ & $0.0157(15)$ & $0.0150(14)$ & $-0.0038(12)$ & $0.0002(11)$ & $-0.0069(12)$ \\
O2 & $0.0179(15)$ & $0.0185(16)$ & $0.0157(14)$ & $-0.0065(12)$ & $0.0021(12)$ & $-0.0055(13)$ \\
O3 & $0.0232(16)$ & $0.0307(19)$ & $0.0185(16)$ & $-0.0101(14)$ & $-0.0013(13)$ & $-0.0100(14)$ \\
O4 & $0.0142(14)$ & $0.0157(15)$ & $0.0179(15)$ & $-0.0041(12)$ & $-0.0013(11)$ & $-0.0104(13)$ \\
O5 & $0.0194(15)$ & $0.0228(17)$ & $0.0223(16)$ & $-0.0053(13)$ & $0.0038(13)$ & $-0.0122(14)$ \\
O6 & $0.0184(15)$ & $0.0146(15)$ & $0.0171(15)$ & $-0.0040(12)$ & $-0.0022(12)$ & $-0.0058(13)$ \\
O7 & $0.0128(14)$ & $0.0170(16)$ & $0.0150(14)$ & $-0.0024(12)$ & $-0.0010(11)$ & $-0.0060(12)$ \\
O8 & $0.0186(15)$ & $0.0155(16)$ & $0.0227(16)$ & $-0.0007(12)$ & $-0.0028(12)$ & $-0.0091(13)$ \\
O9 & $0.0201(16)$ & $0.0316(19)$ & $0.0194(16)$ & $-0.0067(14)$ & $-0.0042(13)$ & $-0.0110(14)$ \\
O10 & $0.0146(14)$ & $0.0169(16)$ & $0.0199(15)$ & $-0.0045(12)$ & $-0.0005(12)$ & $-0.0101(13)$ \\
O11 & $0.0159(15)$ & $0.0214(17)$ & $0.0273(17)$ & $-0.0002(13)$ & $-0.0014(13)$ & $-0.0110(14)$ \\
O12 & $0.0243(16)$ & $0.0219(17)$ & $0.0313(18)$ & $-0.0108(14)$ & $0.0025(14)$ & $-0.0099(15)$ \\
O13 & $0.0171(15)$ & $0.0227(17)$ & $0.0232(16)$ & $-0.0038(13)$ & $0.0018(13)$ & $-0.0098(14)$ \\
N1 & $0.023(2)$ & $0.038(3)$ & $0.022(2)$ & $-0.0038(19)$ & $-0.0028(17)$ & $-0.0087(19)$ \\
N2 & $0.027(2)$ & $0.029(2)$ & $0.024(2)$ & $-0.0071(19)$ & $-0.0013(17)$ & $-0.0067(19)$ \\
N3 & $0.020(2)$ & $0.029(2)$ & $0.046(3)$ & $0.0003(17)$ & $0.0004(18)$ & $-0.026(2)$
\end{tabular}




\begin{tabular}{lllllll}
$\mathrm{N} 4$ & $0.026(2)$ & $0.031(2)$ & $0.041(2)$ & $-0.0094(18)$ & $0.0027(18)$ & $-0.026(2)$ \\
$\mathrm{C} 1$ & $0.061(4)$ & $0.053(4)$ & $0.039(3)$ & $-0.024(3)$ & $-0.005(3)$ & $-0.017(3)$ \\
$\mathrm{C} 2$ & $0.036(3)$ & $0.035(3)$ & $0.020(2)$ & $-0.010(2)$ & $0.004(2)$ & $-0.007(2)$ \\
$\mathrm{C} 3$ & $0.032(3)$ & $0.025(3)$ & $0.025(3)$ & $-0.002(2)$ & $-0.001(2)$ & $-0.001(2)$ \\
$\mathrm{C} 4$ & $0.027(3)$ & $0.033(3)$ & $0.022(2)$ & $-0.004(2)$ & $-0.002(2)$ & $-0.001(2)$ \\
$\mathrm{C} 5$ & $0.028(3)$ & $0.046(4)$ & $0.036(3)$ & $-0.011(2)$ & $-0.006(2)$ & $0.000(3)$ \\
$\mathrm{C} 6$ & $0.024(3)$ & $0.035(3)$ & $0.050(3)$ & $-0.003(2)$ & $-0.008(2)$ & $-0.027(3)$ \\
C7 & $0.021(2)$ & $0.020(2)$ & $0.026(2)$ & $-0.0049(19)$ & $0.0010(18)$ & $-0.014(2)$ \\
C8 & $0.023(2)$ & $0.024(3)$ & $0.026(2)$ & $-0.0022(19)$ & $-0.0022(19)$ & $-0.017(2)$ \\
C9 & $0.022(2)$ & $0.024(2)$ & $0.019(2)$ & $-0.0054(19)$ & $0.0015(18)$ & $-0.011(2)$ \\
C10 & $0.025(2)$ & $0.030(3)$ & $0.043(3)$ & $-0.006(2)$ & $-0.005(2)$ & $-0.021(2)$ \\
O1W & $0.0298(18)$ & $0.036(2)$ & $0.0240(17)$ & $-0.0078(15)$ & $-0.0003(14)$ & $-0.0192(16)$ \\
\hline
\end{tabular}

Geometric parameters $\left(\AA,{ }^{\circ}\right)$

\begin{tabular}{|c|c|c|c|}
\hline Mo1-O3 & $1.702(3)$ & $\mathrm{N} 2-\mathrm{C} 4$ & $1.340(6)$ \\
\hline Mo1-O5 & $1.715(3)$ & $\mathrm{N} 2-\mathrm{H} 2$ & $0.946(10)$ \\
\hline $\mathrm{Mo} 1-\mathrm{O} 2$ & $1.875(3)$ & $\mathrm{N} 3-\mathrm{C} 7$ & $1.332(5)$ \\
\hline Mo1-O4 & $1.982(3)$ & $\mathrm{N} 3-\mathrm{N} 4$ & $1.351(5)$ \\
\hline $\mathrm{Mo} 1-\mathrm{O}^{\mathrm{i}}$ & $2.307(3)$ & $\mathrm{N} 3-\mathrm{H} 3$ & $0.947(10)$ \\
\hline Mo1-O1 & $2.320(3)$ & $\mathrm{N} 4-\mathrm{C} 9$ & $1.328(6)$ \\
\hline Mo1-Mo2 & $3.2062(6)$ & $\mathrm{N} 4-\mathrm{H} 4$ & $0.947(10)$ \\
\hline $\mathrm{Mo} 2-\mathrm{O} 8$ & $1.691(3)$ & $\mathrm{C} 1-\mathrm{C} 2$ & $1.488(7)$ \\
\hline Mo2-O6 & $1.754(3)$ & $\mathrm{C} 1-\mathrm{H} 1 \mathrm{~A}$ & 0.9800 \\
\hline $\mathrm{Mo} 2-\mathrm{O} 7$ & $1.953(3)$ & $\mathrm{C} 1-\mathrm{H} 1 \mathrm{~B}$ & 0.9800 \\
\hline $\mathrm{Mo} 2-\mathrm{O} 4$ & $1.953(3)$ & $\mathrm{C} 1-\mathrm{H} 1 \mathrm{C}$ & 0.9800 \\
\hline $\mathrm{Mo} 2-\mathrm{O} 1$ & $2.146(3)$ & $\mathrm{C} 2-\mathrm{C} 3$ & $1.389(7)$ \\
\hline $\mathrm{Mo} 2-\mathrm{O}^{\mathrm{i}}$ & $2.341(3)$ & $\mathrm{C} 3-\mathrm{C} 4$ & $1.373(7)$ \\
\hline Mo2-Mo3 & $3.2178(5)$ & $\mathrm{C} 3-\mathrm{H} 3 \mathrm{~A}$ & 0.9500 \\
\hline Mo3-O9 & $1.695(3)$ & $\mathrm{C} 4-\mathrm{C} 5$ & $1.488(7)$ \\
\hline Mo3-O11 & $1.695(3)$ & $\mathrm{C} 5-\mathrm{H} 5 \mathrm{~A}$ & 0.9800 \\
\hline Mo3-O10 & $1.915(3)$ & $\mathrm{C} 5-\mathrm{H} 5 \mathrm{~B}$ & 0.9800 \\
\hline Mo3-O7 & 1.998 (3) & $\mathrm{C} 5-\mathrm{H} 5 \mathrm{C}$ & 0.9800 \\
\hline $\mathrm{Mo3}-\mathrm{O} 1$ & $2.341(3)$ & $\mathrm{C} 6-\mathrm{C} 7$ & $1.488(6)$ \\
\hline $\mathrm{Mo} 3-\mathrm{O} 4^{\mathrm{i}}$ & $2.351(3)$ & C6-H6A & 0.9800 \\
\hline Mo4-O12 & $1.692(3)$ & C6-H6B & 0.9800 \\
\hline Mo4-O13 & $1.711(3)$ & $\mathrm{C} 6-\mathrm{H} 6 \mathrm{C}$ & 0.9800 \\
\hline Mo4-O10 & $1.928(3)$ & $\mathrm{C} 7-\mathrm{C} 8$ & $1.379(6)$ \\
\hline $\mathrm{Mo} 4-\mathrm{O} 2$ & $1.943(3)$ & $\mathrm{C} 8-\mathrm{C} 9$ & $1.394(6)$ \\
\hline $\mathrm{Mo} 4-\mathrm{O}^{\mathrm{i}}$ & $2.268(3)$ & $\mathrm{C} 8-\mathrm{H} 8$ & 0.9500 \\
\hline $\mathrm{Mo} 4-\mathrm{O} 1$ & $2.435(3)$ & $\mathrm{C} 9-\mathrm{C} 10$ & $1.483(6)$ \\
\hline $\mathrm{O} 1-\mathrm{Mo} 2^{\mathrm{i}}$ & $2.341(3)$ & $\mathrm{C} 10-\mathrm{H} 10 \mathrm{~A}$ & 0.9800 \\
\hline $\mathrm{O} 4-\mathrm{Mo}^{\mathrm{i}}$ & $2.351(3)$ & $\mathrm{C} 10-\mathrm{H} 10 \mathrm{~B}$ & 0.9800 \\
\hline $\mathrm{O} 6-\mathrm{Mo} 4^{\mathrm{i}}$ & $2.268(3)$ & $\mathrm{C} 10-\mathrm{H} 10 \mathrm{C}$ & 0.9800 \\
\hline $\mathrm{O} 7-\mathrm{Mo1}^{\mathrm{i}}$ & $2.307(3)$ & $\mathrm{O} 1 \mathrm{~W}-\mathrm{H} 1 \mathrm{X}$ & $0.946(10)$ \\
\hline $\mathrm{N} 1-\mathrm{C} 2$ & $1.332(6)$ & $\mathrm{O} 1 \mathrm{~W}-\mathrm{H} 1 \mathrm{Y}$ & $0.944(10)$ \\
\hline $\mathrm{N} 1-\mathrm{N} 2$ & $1.350(5)$ & $\mathrm{O} 2 \mathrm{~W}-\mathrm{O} 3 \mathrm{~W}$ & $0.674(13)$ \\
\hline $\mathrm{N} 1-\mathrm{H} 1$ & $0.943(10)$ & & \\
\hline
\end{tabular}




\begin{tabular}{|c|c|c|c|}
\hline $\mathrm{O} 3-\mathrm{Mo} 1-\mathrm{O} 5$ & $104.71(14)$ & $\mathrm{O} 12-\mathrm{Mo} 4-\mathrm{O} 1$ & $162.52(12)$ \\
\hline $\mathrm{O} 3-\mathrm{Mo} 1-\mathrm{O} 2$ & $102.12(13)$ & $\mathrm{O} 13-\mathrm{Mo} 4-\mathrm{O} 1$ & $91.62(11)$ \\
\hline $\mathrm{O} 5-\mathrm{Mo} 1-\mathrm{O} 2$ & $100.72(13)$ & $\mathrm{O} 10-\mathrm{Mo} 4-\mathrm{O} 1$ & $74.97(10)$ \\
\hline $\mathrm{O} 3-\mathrm{Mo} 1-\mathrm{O} 4$ & $99.75(12)$ & $\mathrm{O} 2-\mathrm{Mo} 4-\mathrm{O} 1$ & $73.62(10)$ \\
\hline $\mathrm{O} 5-\mathrm{Mo1}-\mathrm{O} 4$ & $97.01(13)$ & $\mathrm{O} 6-\mathrm{i}-\mathrm{Mo} 4-\mathrm{O} 1$ & $70.01(9)$ \\
\hline $\mathrm{O} 2-\mathrm{Mo1}-\mathrm{O} 4$ & $147.18(11)$ & Mo2-O1-Mo1 & $91.68(9)$ \\
\hline $\mathrm{O} 3-\mathrm{Mo1}-\mathrm{O}^{\mathrm{i}}$ & $89.69(12)$ & $\mathrm{Mo} 2-\mathrm{O} 1-\mathrm{Mo} 2^{\mathrm{i}}$ & $104.01(10)$ \\
\hline $\mathrm{O} 5-\mathrm{Mo} 1-\mathrm{O}^{\mathrm{i}}$ & $163.56(12)$ & $\mathrm{Mo} 1-\mathrm{O} 1-\mathrm{Mo} 2^{\mathrm{i}}$ & $97.04(9)$ \\
\hline $\mathrm{O} 2-\mathrm{Mo} 1-\mathrm{O} 7^{\mathrm{i}}$ & $83.57(11)$ & $\mathrm{Mo2}-\mathrm{O} 1-\mathrm{Mo} 3$ & $91.54(10)$ \\
\hline $\mathrm{O} 4-\mathrm{Mo} 1-\mathrm{O}^{\mathrm{i}}$ & $72.34(10)$ & Mo1-O1—Mo3 & $163.07(12)$ \\
\hline $\mathrm{O} 3-\mathrm{Mo1}-\mathrm{O} 1$ & $161.88(12)$ & $\mathrm{Mo} 2 \mathrm{i}-\mathrm{O} 1-\mathrm{Mo} 3$ & $98.29(9)$ \\
\hline $\mathrm{O} 5-\mathrm{Mo1}-\mathrm{O} 1$ & $93.03(11)$ & Mo2-O1-Mo4 & $163.99(13)$ \\
\hline $\mathrm{O} 2-\mathrm{Mo} 1-\mathrm{O} 1$ & $77.65(10)$ & Mo1-O1-Mo4 & $86.35(9)$ \\
\hline $\mathrm{O} 4-\mathrm{Mo1}-\mathrm{O} 1$ & $74.01(10)$ & $\mathrm{Mo2}-\mathrm{O} 1-\mathrm{Mo} 4$ & $91.99(9)$ \\
\hline $\mathrm{O} 7 \mathrm{i}-\mathrm{Mo1}-\mathrm{O} 1$ & $72.23(9)$ & Mo3-O1-Mo4 & $85.98(8)$ \\
\hline $\mathrm{O} 3-\mathrm{Mo} 1-\mathrm{Mo} 2$ & $134.82(10)$ & Mo1-O2-Mo4 & $116.94(14)$ \\
\hline $\mathrm{O} 5-\mathrm{Mo1}-\mathrm{Mo2}$ & $84.85(10)$ & Mo2-O4-Mo1 & $109.13(12)$ \\
\hline $\mathrm{O} 2-\mathrm{Mo1}-\mathrm{Mo2}$ & $119.63(8)$ & $\mathrm{Mo} 2-\mathrm{O} 4-\mathrm{Mo}^{\mathrm{i}}$ & $110.39(11)$ \\
\hline $\mathrm{O} 4-\mathrm{Mo1}-\mathrm{Mo2}$ & $35.14(7)$ & $\mathrm{Mo} 1-\mathrm{O} 4-\mathrm{Mo}^{\mathrm{i}}$ & $103.30(11)$ \\
\hline $\mathrm{O} 7 \mathrm{i}-\mathrm{Mo} 1-\mathrm{Mo} 2$ & $79.31(7)$ & $\mathrm{Mo} 2-\mathrm{O} 6-\mathrm{Mo}^{\mathrm{i}}$ & $116.79(14)$ \\
\hline $\mathrm{O} 1-\mathrm{Mo1}-\mathrm{Mo2}$ & $41.99(7)$ & Mo2-O7-Mo3 & $109.07(13)$ \\
\hline $\mathrm{O} 8-\mathrm{Mo} 2-\mathrm{O} 6$ & $105.54(13)$ & $\mathrm{Mo} 2-\mathrm{O} 7-\mathrm{Mo}^{\mathrm{i}}$ & $109.84(11)$ \\
\hline $\mathrm{O} 8-\mathrm{Mo} 2-\mathrm{O} 7$ & $101.63(12)$ & $\mathrm{Mo3}-\mathrm{O} 7-\mathrm{Mol}^{\mathrm{i}}$ & $104.33(12)$ \\
\hline O6-Мo2-O7 & $96.27(12)$ & $\mathrm{Mo3}-\mathrm{O} 10-\mathrm{Mo} 4$ & $115.92(13)$ \\
\hline $\mathrm{O} 8-\mathrm{Mo} 2-\mathrm{O} 4$ & $99.93(12)$ & $\mathrm{C} 2-\mathrm{N} 1-\mathrm{N} 2$ & $108.8(4)$ \\
\hline $\mathrm{O} 6-\mathrm{Mo} 2-\mathrm{O} 4$ & $97.02(11)$ & $\mathrm{C} 2-\mathrm{N} 1-\mathrm{H} 1$ & $133(3)$ \\
\hline $\mathrm{O} 7-\mathrm{Mo} 2-\mathrm{O} 4$ & $150.55(11)$ & $\mathrm{N} 2-\mathrm{N} 1-\mathrm{H} 1$ & $118(3)$ \\
\hline $\mathrm{O} 8-\mathrm{Mo} 2-\mathrm{O} 1$ & $97.27(12)$ & $\mathrm{C} 4-\mathrm{N} 2-\mathrm{N} 1$ & $108.9(4)$ \\
\hline $\mathrm{O} 6-\mathrm{Mo} 2-\mathrm{O} 1$ & $157.19(11)$ & $\mathrm{C} 4-\mathrm{N} 2-\mathrm{H} 2$ & $128(3)$ \\
\hline $\mathrm{O} 7-\mathrm{Mo} 2-\mathrm{O} 1$ & $78.87(10)$ & $\mathrm{N} 1-\mathrm{N} 2-\mathrm{H} 2$ & $123(3)$ \\
\hline $\mathrm{O} 4-\mathrm{Mo} 2-\mathrm{O} 1$ & $78.71(10)$ & $\mathrm{C} 7-\mathrm{N} 3-\mathrm{N} 4$ & $109.1(4)$ \\
\hline $\mathrm{O} 8-\mathrm{Mo} 2-\mathrm{O} 1^{\mathrm{i}}$ & $173.17(12)$ & $\mathrm{C} 7-\mathrm{N} 3-\mathrm{H} 3$ & $133(3)$ \\
\hline $\mathrm{O} 6-\mathrm{Mo} 2-\mathrm{O} 1^{\mathrm{i}}$ & $81.21(11)$ & $\mathrm{N} 4-\mathrm{N} 3-\mathrm{H} 3$ & $117(3)$ \\
\hline $\mathrm{O} 7-\mathrm{Mo} 2-\mathrm{O} 1^{\mathrm{i}}$ & $78.31(10)$ & $\mathrm{C} 9-\mathrm{N} 4-\mathrm{N} 3$ & $109.5(4)$ \\
\hline $\mathrm{O} 4-\mathrm{Mo} 2-\mathrm{O}^{\mathrm{i}}$ & $77.92(10)$ & $\mathrm{C} 9-\mathrm{N} 4-\mathrm{H} 4$ & $128(3)$ \\
\hline $\mathrm{O} 1-\mathrm{Mo} 2-\mathrm{O} 1^{\mathrm{i}}$ & $75.99(10)$ & $\mathrm{N} 3-\mathrm{N} 4-\mathrm{H} 4$ & $122(3)$ \\
\hline $\mathrm{O} 8-\mathrm{Mo} 2-\mathrm{Mo} 1$ & $88.75(10)$ & $\mathrm{C} 2-\mathrm{C} 1-\mathrm{H} 1 \mathrm{~A}$ & 109.5 \\
\hline $\mathrm{O} 6-\mathrm{Mo} 2-\mathrm{Mo1}$ & $132.75(9)$ & $\mathrm{C} 2-\mathrm{C} 1-\mathrm{H} 1 \mathrm{~B}$ & 109.5 \\
\hline $\mathrm{O} 7-\mathrm{Mo2}-\mathrm{Mo1}$ & $125.17(8)$ & $\mathrm{H} 1 \mathrm{~A}-\mathrm{C} 1-\mathrm{H} 1 \mathrm{~B}$ & 109.5 \\
\hline $\mathrm{O} 4-\mathrm{Mo} 2-\mathrm{Mo} 1$ & $35.73(8)$ & $\mathrm{C} 2-\mathrm{C} 1-\mathrm{H} 1 \mathrm{C}$ & 109.5 \\
\hline $\mathrm{O} 1-\mathrm{Mo} 2-\mathrm{Mo} 1$ & $46.33(7)$ & $\mathrm{H} 1 \mathrm{~A}-\mathrm{C} 1-\mathrm{H} 1 \mathrm{C}$ & 109.5 \\
\hline $\mathrm{O} 1{ }^{\mathrm{i}}-\mathrm{Mo} 2-\mathrm{Mo} 1$ & $85.79(7)$ & $\mathrm{H} 1 \mathrm{~B}-\mathrm{C} 1-\mathrm{H} 1 \mathrm{C}$ & 109.5 \\
\hline $\mathrm{O} 8-\mathrm{Mo} 2-\mathrm{Mo3}$ & $89.31(9)$ & $\mathrm{N} 1-\mathrm{C} 2-\mathrm{C} 3$ & $107.8(4)$ \\
\hline $\mathrm{O} 6-\mathrm{Mo2}-\mathrm{Mo3}$ & $132.20(9)$ & $\mathrm{N} 1-\mathrm{C} 2-\mathrm{C} 1$ & $121.8(5)$ \\
\hline $\mathrm{O} 7-\mathrm{Mo2}-\mathrm{Mo3}$ & $35.93(8)$ & $\mathrm{C} 3-\mathrm{C} 2-\mathrm{C} 1$ & $130.4(5)$ \\
\hline $\mathrm{O} 4-\mathrm{Mo} 2-\mathrm{Mo3}$ & $125.36(8)$ & $\mathrm{C} 4-\mathrm{C} 3-\mathrm{C} 2$ & $106.6(4)$ \\
\hline $\mathrm{O} 1-\mathrm{Mo} 2-\mathrm{Mo} 3$ & $46.65(7)$ & $\mathrm{C} 4-\mathrm{C} 3-\mathrm{H} 3 \mathrm{~A}$ & 126.7 \\
\hline
\end{tabular}




\begin{tabular}{|c|c|c|c|}
\hline $\mathrm{O} 1{ }^{\mathrm{i}}-\mathrm{Mo} 2-\mathrm{Mo} 3$ & $86.76(6)$ & $\mathrm{C} 2-\mathrm{C} 3-\mathrm{H} 3 \mathrm{~A}$ & 126.7 \\
\hline Mo1-Mo2-Mo3 & $91.724(14)$ & $\mathrm{N} 2-\mathrm{C} 4-\mathrm{C} 3$ & $107.8(4)$ \\
\hline $\mathrm{O} 9-\mathrm{Mo} 3-\mathrm{O} 11$ & $105.85(14)$ & $\mathrm{N} 2-\mathrm{C} 4-\mathrm{C} 5$ & $121.2(5)$ \\
\hline $\mathrm{O} 9-\mathrm{Mo} 3-\mathrm{O} 10$ & $100.45(13)$ & $\mathrm{C} 3-\mathrm{C} 4-\mathrm{C} 5$ & $130.9(5)$ \\
\hline $\mathrm{O} 11-\mathrm{Mo} 3-\mathrm{O} 10$ & $101.76(13)$ & $\mathrm{C} 4-\mathrm{C} 5-\mathrm{H} 5 \mathrm{~A}$ & 109.5 \\
\hline $\mathrm{O} 9-\mathrm{Mo3}-\mathrm{O} 7$ & $100.71(12)$ & $\mathrm{C} 4-\mathrm{C} 5-\mathrm{H} 5 \mathrm{~B}$ & 109.5 \\
\hline $\mathrm{O} 11-\mathrm{Mo3}-\mathrm{O} 7$ & $97.58(12)$ & $\mathrm{H} 5 \mathrm{~A}-\mathrm{C} 5-\mathrm{H} 5 \mathrm{~B}$ & 109.5 \\
\hline $\mathrm{O} 10-\mathrm{Mo} 3-\mathrm{O} 7$ & $146.06(11)$ & $\mathrm{C} 4-\mathrm{C} 5-\mathrm{H} 5 \mathrm{C}$ & 109.5 \\
\hline $\mathrm{O} 9-\mathrm{Mo} 3-\mathrm{O} 1$ & $159.60(12)$ & $\mathrm{H} 5 \mathrm{~A}-\mathrm{C} 5-\mathrm{H} 5 \mathrm{C}$ & 109.5 \\
\hline $\mathrm{O} 11-\mathrm{Mo} 3-\mathrm{O} 1$ & $94.36(12)$ & $\mathrm{H} 5 \mathrm{~B}-\mathrm{C} 5-\mathrm{H} 5 \mathrm{C}$ & 109.5 \\
\hline $\mathrm{O} 10-\mathrm{Mo} 3-\mathrm{O} 1$ & $77.54(10)$ & $\mathrm{C} 7-\mathrm{C} 6-\mathrm{H} 6 \mathrm{~A}$ & 109.5 \\
\hline $\mathrm{O} 7-\mathrm{Mo} 3-\mathrm{O} 1$ & $73.40(10)$ & $\mathrm{C} 7-\mathrm{C} 6-\mathrm{H} 6 \mathrm{~B}$ & 109.5 \\
\hline $\mathrm{O} 9-\mathrm{Mo} 3-\mathrm{O} 4^{\mathrm{i}}$ & $88.84(12)$ & $\mathrm{H} 6 \mathrm{~A}-\mathrm{C} 6-\mathrm{H} 6 \mathrm{~B}$ & 109.5 \\
\hline $\mathrm{O} 11-\mathrm{Mo} 3-\mathrm{O} 4^{\mathrm{i}}$ & $163.17(11)$ & $\mathrm{C} 7-\mathrm{C} 6-\mathrm{H} 6 \mathrm{C}$ & 109.5 \\
\hline $\mathrm{O} 10-\mathrm{Mo} 3-\mathrm{O}^{\mathrm{i}}$ & $83.12(10)$ & $\mathrm{H} 6 \mathrm{~A}-\mathrm{C} 6-\mathrm{H} 6 \mathrm{C}$ & 109.5 \\
\hline $\mathrm{O} 7-\mathrm{Mo} 3-\mathrm{O} 4^{\mathrm{i}}$ & $71.11(10)$ & $\mathrm{H} 6 \mathrm{~B}-\mathrm{C} 6-\mathrm{H} 6 \mathrm{C}$ & 109.5 \\
\hline $\mathrm{O} 1-\mathrm{Mo} 3-\mathrm{O} 4^{\mathrm{i}}$ & $70.77(9)$ & $\mathrm{N} 3-\mathrm{C} 7-\mathrm{C} 8$ & $107.4(4)$ \\
\hline O9-Mo3-Mo2 & $135.69(10)$ & $\mathrm{N} 3-\mathrm{C} 7-\mathrm{C} 6$ & $121.8(4)$ \\
\hline O11-Mo3-Mo2 & $85.49(10)$ & $\mathrm{C} 8-\mathrm{C} 7-\mathrm{C} 6$ & $130.8(4)$ \\
\hline $\mathrm{O} 10-\mathrm{Mo} 3-\mathrm{Mo} 2$ & $119.34(8)$ & $\mathrm{C} 7-\mathrm{C} 8-\mathrm{C} 9$ & $107.0(4)$ \\
\hline $\mathrm{O} 7-\mathrm{Mo3}-\mathrm{Mo} 2$ & $35.00(7)$ & $\mathrm{C} 7-\mathrm{C} 8-\mathrm{H} 8$ & 126.5 \\
\hline $\mathrm{O} 1-\mathrm{Mo3}-\mathrm{Mo} 2$ & $41.81(7)$ & $\mathrm{C} 9-\mathrm{C} 8-\mathrm{H} 8$ & 126.5 \\
\hline $\mathrm{O} 4$ - $-\mathrm{Mo} 3-\mathrm{Mo} 2$ & $78.16(6)$ & $\mathrm{N} 4-\mathrm{C} 9-\mathrm{C} 8$ & $106.9(4)$ \\
\hline $\mathrm{O} 12-\mathrm{Mo} 4-\mathrm{O} 13$ & $105.75(14)$ & $\mathrm{N} 4-\mathrm{C} 9-\mathrm{C} 10$ & $121.9(4)$ \\
\hline $\mathrm{O} 12-\mathrm{Mo} 4-\mathrm{O} 10$ & $103.91(13)$ & $\mathrm{C} 8-\mathrm{C} 9-\mathrm{C} 10$ & $131.2(4)$ \\
\hline $\mathrm{O} 13-\mathrm{Mo} 4-\mathrm{O} 10$ & $97.77(13)$ & $\mathrm{C} 9-\mathrm{C} 10-\mathrm{H} 10 \mathrm{~A}$ & 109.5 \\
\hline $\mathrm{O} 12-\mathrm{Mo} 4-\mathrm{O} 2$ & $101.63(13)$ & C9- $10-\mathrm{H} 10 \mathrm{~B}$ & 109.5 \\
\hline $\mathrm{O} 13-\mathrm{Mo} 4-\mathrm{O} 2$ & $97.49(13)$ & $\mathrm{H} 10 \mathrm{~A}-\mathrm{C} 10-\mathrm{H} 10 \mathrm{~B}$ & 109.5 \\
\hline $\mathrm{O} 10-\mathrm{Mo} 4-\mathrm{O} 2$ & $145.29(11)$ & $\mathrm{C} 9-\mathrm{C} 10-\mathrm{H} 10 \mathrm{C}$ & 109.5 \\
\hline $\mathrm{O} 12-\mathrm{Mo} 4-\mathrm{O}^{\mathrm{i}}$ & $92.59(12)$ & $\mathrm{H} 10 \mathrm{~A}-\mathrm{C} 10-\mathrm{H} 10 \mathrm{C}$ & 109.5 \\
\hline $\mathrm{O} 13-\mathrm{Mo} 4-\mathrm{O}^{\mathrm{i}}$ & $161.61(12)$ & $\mathrm{H} 10 \mathrm{~B}-\mathrm{C} 10-\mathrm{H} 10 \mathrm{C}$ & 109.5 \\
\hline $\mathrm{O} 10-\mathrm{Mo} 4-\mathrm{O}^{\mathrm{i}}$ & $78.80(10)$ & $\mathrm{H} 1 \mathrm{X}-\mathrm{O} 1 \mathrm{~W}-\mathrm{H} 1 \mathrm{Y}$ & $109.9(16)$ \\
\hline $\mathrm{O} 2-\mathrm{Mo} 4-\mathrm{O}^{\mathrm{i}}$ & $76.95(11)$ & & \\
\hline $\mathrm{O} 3-\mathrm{Mo} 1-\mathrm{O} 2-\mathrm{Mo} 4$ & $177.15(15)$ & $\mathrm{N} 2-\mathrm{N} 1-\mathrm{C} 2-\mathrm{C} 1$ & $-178.3(4)$ \\
\hline $\mathrm{O} 5-\mathrm{Mo} 1-\mathrm{O} 2-\mathrm{Mo} 4$ & $69.40(17)$ & $\mathrm{N} 1-\mathrm{C} 2-\mathrm{C} 3-\mathrm{C} 4$ & $0.4(5)$ \\
\hline $\mathrm{O} 4-\mathrm{Mo} 1-\mathrm{O} 2-\mathrm{Mo} 4$ & $-52.1(3)$ & $\mathrm{C} 1-\mathrm{C} 2-\mathrm{C} 3-\mathrm{C} 4$ & $179.0(5)$ \\
\hline $\mathrm{O} 7-\mathrm{i}-\mathrm{Mo} 1-\mathrm{O} 2-\mathrm{Mo} 4$ & $-94.55(15)$ & $\mathrm{N} 1-\mathrm{N} 2-\mathrm{C} 4-\mathrm{C} 3$ & $1.4(5)$ \\
\hline $\mathrm{O} 1-\mathrm{Mo} 1-\mathrm{O} 2-\mathrm{Mo} 4$ & $-21.39(13)$ & $\mathrm{N} 1-\mathrm{N} 2-\mathrm{C} 4-\mathrm{C} 5$ & $-179.1(4)$ \\
\hline $\mathrm{Mo} 2-\mathrm{Mo} 1-\mathrm{O} 2-\mathrm{Mo} 4$ & $-20.75(18)$ & $\mathrm{C} 2-\mathrm{C} 3-\mathrm{C} 4-\mathrm{N} 2$ & $-1.1(5)$ \\
\hline $\mathrm{O} 8-\mathrm{Mo} 2-\mathrm{O} 6-\mathrm{Mo}^{4}$ & $178.38(13)$ & $\mathrm{C} 2-\mathrm{C} 3-\mathrm{C} 4-\mathrm{C} 5$ & $179.4(5)$ \\
\hline $\mathrm{O} 7-\mathrm{Mo} 2-\mathrm{O} 6-\mathrm{Mo}^{\mathrm{i}}$ & $-77.67(14)$ & $\mathrm{N} 4-\mathrm{N} 3-\mathrm{C} 7-\mathrm{C} 8$ & $-0.1(5)$ \\
\hline $\mathrm{O} 4-\mathrm{Mo} 2-\mathrm{O} 6-\mathrm{Mo} 4^{\mathrm{i}}$ & $75.99(14)$ & $\mathrm{N} 4-\mathrm{N} 3-\mathrm{C} 7-\mathrm{C} 6$ & $-178.6(4)$ \\
\hline $\mathrm{O} 1-\mathrm{Mo} 2-\mathrm{O} 6-\mathrm{Mo}^{\mathrm{i}}$ & $-1.5(4)$ & $\mathrm{N} 3-\mathrm{C} 7-\mathrm{C} 8-\mathrm{C} 9$ & $0.4(5)$ \\
\hline $\mathrm{O} 1^{\mathrm{i}}-\mathrm{Mo} 2-\mathrm{O} 6-\mathrm{Mo}^{\mathrm{i}}$ & $-0.57(12)$ & $\mathrm{C} 6-\mathrm{C} 7-\mathrm{C} 8-\mathrm{C} 9$ & $178.7(5)$ \\
\hline $\mathrm{Mo} 1-\mathrm{Mo} 2-\mathrm{O} 6-\mathrm{Mo}^{\mathrm{i}}$ & $75.31(16)$ & $\mathrm{N} 3-\mathrm{N} 4-\mathrm{C} 9-\mathrm{C} 8$ & $0.5(5)$ \\
\hline $\mathrm{Mo} 3-\mathrm{Mo} 2-\mathrm{O} 6-\mathrm{Mo}^{\mathrm{i}}$ & $-78.01(15)$ & $\mathrm{N} 3-\mathrm{N} 4-\mathrm{C} 9-\mathrm{C} 10$ & $179.3(4)$ \\
\hline $\mathrm{C} 2-\mathrm{N} 1-\mathrm{N} 2-\mathrm{C} 4$ & $-1.1(5)$ & $\mathrm{C} 7-\mathrm{C} 8-\mathrm{C} 9-\mathrm{N} 4$ & $-0.6(5)$ \\
\hline
\end{tabular}




$\mathrm{C} 7-\mathrm{N} 3-\mathrm{N} 4-\mathrm{C} 9 \quad-0.2(5) \quad \mathrm{C} 7-\mathrm{C} 8-\mathrm{C} 9-\mathrm{C} 10 \quad-179.2(5)$

$\mathrm{N} 2-\mathrm{N} 1-\mathrm{C} 2-\mathrm{C} 3 \quad 0.4(5)$

Symmetry code: (i) $-x+1,-y+1,-z+1$.

Hydrogen-bond geometry $\left(A,{ }^{\circ}\right)$

\begin{tabular}{lllll}
\hline$D-\mathrm{H} \cdots A$ & $D-\mathrm{H}$ & $\mathrm{H} \cdots A$ & $D \cdots A$ & $D-\mathrm{H} \cdots A$ \\
\hline $\mathrm{N} 1-\mathrm{H} 1 \cdots \mathrm{O} 2 W^{\text {ii }}$ & 0.94 & 1.77 & $2.689(7)$ & 164 \\
$\mathrm{~N} 2-\mathrm{H} 2 \cdots \mathrm{O} 1 W$ & 0.95 & 1.84 & $2.776(5)$ & 171 \\
$\mathrm{~N} 3-\mathrm{H} 3 \cdots \mathrm{O} 13^{\mathrm{iii}}$ & 0.95 & 2.28 & $2.869(5)$ & 120 \\
$\mathrm{~N} 4-\mathrm{H} 4 \cdots \mathrm{O} 10$ & 0.95 & 1.93 & $2.801(4)$ & 152 \\
$\mathrm{O} 1 W-\mathrm{H} 1 X^{\cdots \mathrm{O} 5^{\text {iv }}}$ & 0.95 & 1.88 & $2.785(4)$ & 160 \\
$\mathrm{O} 1 W-\mathrm{H} 1 Y \cdots \mathrm{O} 3$ & 0.94 & 1.91 & $2.848(4)$ & 172
\end{tabular}

Symmetry codes: (ii) $x, y, z+1$; (iii) $-x,-y+1,-z+1$; (iv) $-x+1,-y+1,-z+2$. 